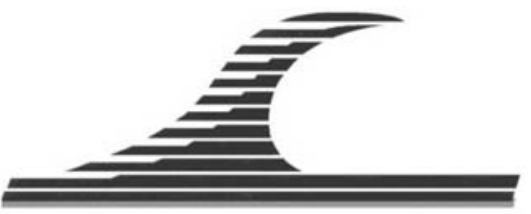

\title{
Valorisation des sédiments fluviaux traités en vue d'une utilisation en génie civil
}

\author{
Zhibo DUAN ${ }^{1}$, Zoubeir LAFHAJ ${ }^{1}$, \\ Imen BEL HADJ ALI ${ }^{1}$, Sébastien DUCELLIER ${ }^{1}$
}

1. Laboratoire de Mécanique de Lille, CNRS UMR 8107, Boulevard Paul Langevin, 59655, Villeneuve d'Ascq Cedex, France. Ecole Centrale de Lille, Cité Scientifique, 59651, Villeneuve d'Ascq Cedex, France. Zoubeir.lafhaj@ec-lille.fr

\section{Résumé :}

L'objectif de cet article est d'étudier la possibilité de valoriser, après traitement, des sédiments fluviaux pollués, prélevés de la région de Dampremy en Belgique, dans les matériaux de génie civil. L'article se décompose en trois parties : la première partie est consacrée à la présentation du procédé de traitement qui a été retenu pour détruire les polluants organiques et stabiliser les polluants inorganiques contenus dans les sédiments. La deuxième partie développe la démarche scientifique et la méthodologie expérimentale retenue. Enfin, la troisième partie, est consacrée à la présentation et à l'analyse des principaux résultats de valorisation des sédiments traités dans les matériaux de génie civil. La formulation retenue se compose de $75 \%$ de sédiments traités, de sable, de ciment et de la chaux vive. Deux voies de valorisation possibles : en matériaux autocompactant et en couche de forme.

Soumis le 18 janvier 2011, accepté le 16 décembre 2011, en ligne le 30 septembre 2013.

La seule version examinée est celle écrite en français. La ou les autres versions n'étant pas examinées par le comité de rédaction de la revue, sont donc publiées sous l'entière responsabilité du ou des auteurs.

Pour citer cet article :

DUAN Z., LAFHAJ Z., BEL HADJ ALI I., DUCELLIER S. (2013). Valorisation des sédiments fluviaux traités en vue d'une utilisation en génie civil. Revue Paralia, Vol. 6, pp 5.1-5.12.

DOI:10.5150/revue-paralia.2013.005 (disponible en ligne - http://www.paralia.fr - available online) 


\section{2 : Revue Paralia - Vol. 6 (2013)}

\section{Introduction}

Disposer de tirants d'eau suffisants et sécuritaires dans les ports, les estuaires et les cours d'eau est indispensable au bon fonctionnement du commerce par voies maritimes et fluviales. Des opérations de dragage des sédiments sont donc nécessaires pour entretenir, approfondir ou aménager les sites portuaires. Mais, ces sédiments contiennent deux types de substances polluantes: des substances organiques (HAP, $\mathrm{PCB}, \ldots)$ et des métaux lourds $(\mathrm{Pb}, \mathrm{Cd}, \mathrm{Zn}, \mathrm{Cu}, \mathrm{As}, \ldots)$ qui, en dépassant certains seuils, présentent un danger pour les humains, la faune et la flore.

Des règles environnementales strictes, ont du être mises au point, depuis la signature de la convention de Londres (LONDON CONVENTION, 1972) pour règlementer la gestion des sédiments dans le monde. Plus de 50 millions de $\mathrm{m}^{3}$ de ces sédiments sont dragués chaque année en France où $10 \%$ à 15\% sont pollués. (ALZIEU, 1999; BOUTOUIL \& LEVACHER, 2000 ; LAFHAJ et al., 2007 ; DUAN et al., 2008). Ces règles contraignent les gestionnaires des opérations de dragage à trouver (OJ, 2000), outre le relargage en mer et l'épandage, de nouvelles solutions en respect avec l'environnement. En effet, les sédiments sont concernés par une double problématique : un volume très important à draguer et une pollution aigue. Ainsi, des chercheurs et des industriels se sont intéressés à la problématique des sédiments pollués et à la mise au point de procédés de traitement. L'entreprise Solvay a développé un procédé : Novosol ${ }^{\circledR}$ qui permet de stabiliser les métaux lourds par phosphatation et de détruire les composés organiques par calcination (SOLVAY, 2002).

Pour rendre les opérations de dragage et de traitement rentables, la valorisation des sédiments s'est avérée indispensable. Plusieurs chercheurs se sont intéressés à la valorisation des sédiments pollués ou non dans le domaine du génie civil : fabrication de briques (WENG et al., 2003 ; LAFHAJ et al., 2008), en granulats artificiels (BRAKNI et al., 2007), en construction routière (KAMALI et al., 2008 ; ZENTAR et al., 2008 ; DUBOIS et al., 2009 ; SCORDIA et al., 2008) et en matériaux cimentaires (AGOSTINI et al., 2007).

Le travail présenté dans cet article a pour objectif d'étudier la possibilité de valoriser des sédiments fluviaux pollués en vue d'une utilisation dans des matériaux de génie civil. Dans un premier temps, une description du procédé de traitement utilisé sera présentée. Ensuite, le protocole expérimental suivi pour l'étude de la valorisation des sédiments traités et les principaux résultats expérimentaux obtenus, seront exposés et analysés.

\section{Le procédé de traitement Novosol ${ }^{\circledR}$}

Le procédé s'appuie sur l'aptitude d'un minéral naturel l'hydroxyapatite, à fixer les métaux lourds. Contrairement à la plupart des techniques de traitement qui visent à éliminer ces polluants, le procédé Novosol ${ }^{\circledR}$ se contente de réduire leur solubilité. Le traitement se décompose en trois étapes (SOLVAY, 2002). La première étape permet la 
stabilisation des métaux lourds par piégeage dans de l'hydroxypaptite $\left(\mathrm{Ca}_{5}\left(\mathrm{PO}_{4}\right)^{3} \mathrm{OH}\right)$. Elle consiste à ajouter de 2 à $3.5 \%$ d'acide phosphorique $\left(\mathrm{H}_{3} \mathrm{PO}_{4}\right)$ qui réagit avec le Calcium présent dans les sédiments. Cette réaction forme l'apatite. C'est un minéral à base de phosphate. Les métaux lourds présents dans les sédiments sont donc stabilisés dans la structure cristalline ainsi créée (l'apatite de calcium $\left.\mathrm{Ca}_{3}\left(\mathrm{PO}_{4}\right)^{2}\right)$. L'efficacité de ce mécanisme de stabilisation a été prouvée par de nombreuses études (LAPERCHE et al., 1996 ; CRANNELL et al., 2000, KRIBI et al., 2004). Les sédiments phosphatés sont ensuite disposés dans des lits de séchage où ils sont laissés jusqu'à obtenir un degré de saturation adéquat avant la dernière phase du procédé.

La troisième phase permet la destruction des composés organiques (Hydrocarbures Aromatiques Polycycliques, Poly-Chloro-Biphényles, dioxines et pesticides) par calcination à une température supérieure à $650^{\circ} \mathrm{C}$ dans un four rotatif.

Les sédiments obtenus à l'issue de ce traitement, sont friables et leurs volumes sont moins importants. Les gaz émis lors de la réaction (essentiellement du $\mathrm{CO}_{2}$ et du $\mathrm{H}_{2} \mathrm{~S}$ et des traces de métaux lourds) sont collectés et traités par ajout de charbon et de bicarbonate de sodium (SAMARA et al., 2008). Pour plus d'informations concernant ce procédé de traitement, se référer à SOLVAY (2002) et AUBERT (2002).

Les sédiments traités, peuvent être décrits, comme étant une poudre, sans odeur, à particules fines de poids volumique $\left(\approx 2.8 \mathrm{~g} \mathrm{~cm}^{-3}\right)$ comparable à celui des argiles $(2.6-$ $2.7 \mathrm{~g} \mathrm{~cm}^{-3}$ ). Les granulats, de ces sédiments traités, sont généralement de forme angulaire à circulaire et principalement composés d'agglomérats de fines de surface spécifique ( $\mathrm{Ss} \approx 4 \mathrm{~m}^{2} \mathrm{~g}^{-1}$ ), possédant ainsi, l'aptitude d'absorber de grandes quantités d'eau (SAMARA et al., 2008).

L'innocuité environnementale de ces sédiments traités a fait l'objet de plusieurs études (NGUYEN, 2008 ; KRIBI et al., 2004).

Concernant l'étude de l'efficacité du procédé, la lixiviation des métaux lourds a été effectuée selon deux procédures différentes : la norme française (AFNOR, 1998), où les sédiments ont été lessivé à l'aide d'eau distillée et la procédure de lixiviation américaine TCLP Toxicity Characteristic Leaching Procedure (USEPA, 1986 ; USEPA, 1992), où l'acide acétique est utilisé comme solution de lessivage.

\section{Matériaux et méthodologie}

\subsection{Caractérisation des sédiments}

Les sédiments utilisés dans cette étude ont été prélevés dans la région de Dampremy en Belgique (du canal reliant Chaleroi à Bruxelles), et traités par le procédé Novosol ${ }^{\circledR}$, sur l'unité pilote installée à Dampremy. Les caractéristiques physico-chimiques, environnementales et minéralogiques de ces sédiments ont été déterminées dans les travaux de Scordia (SCORDIA et al., 2008). La composition chimique des sédiments traités est présentée dans le tableau 1. D'après la courbe granulométrique indiquée sur la 
figure 1 , le coefficient d'uniformité $C_{u}$ est égal à 6 et le coefficient de courbure $C_{c}$ est égal à 2. Ainsi, la granulométrie des sédiments traités est étalée et bien graduée. La composition minéralogique et les propriétés physiques sont données dans le tableau 2. Selon la classification (GTR, 2000), ces sédiments traités appartiennent à la classe A1, qui regroupe les limons peu plastiques, les silts alluvionnaires, les lœss, les sables fins peu pollués et les arènes peu plastiques.

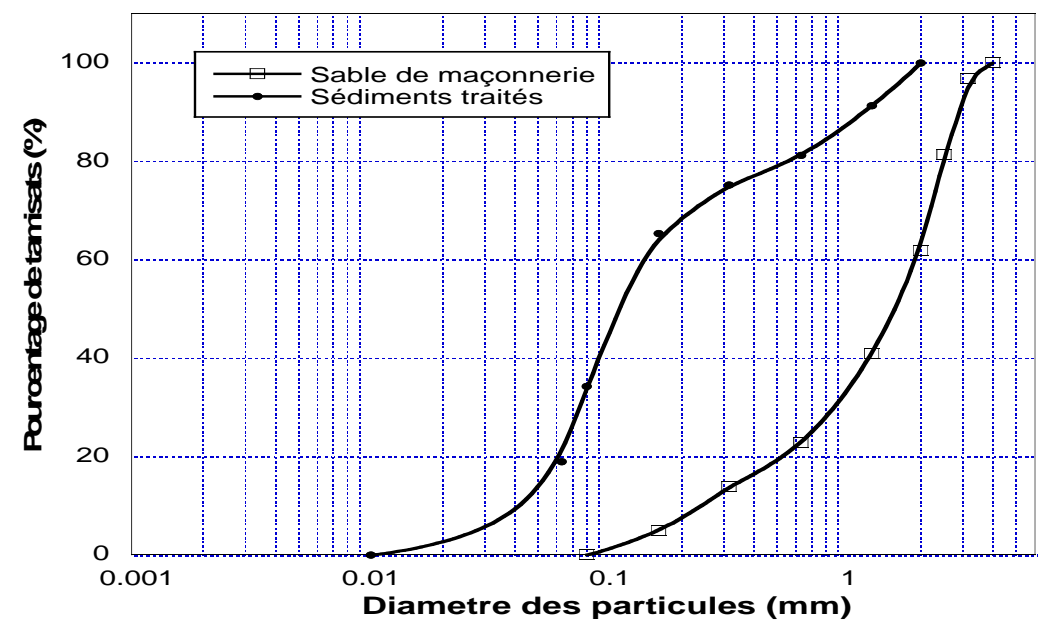

Figure 1. Distribution granulométrique des sédiments traités et du sable utilisé (SCORDIA et al., 2008).

Tableau 1. Composition chimique des sédiments traités, (NGUYEN, 2008).

\begin{tabular}{lllllllll}
\hline $\mathrm{SiO}_{2}$ & $\mathrm{Al}_{2} \mathrm{O}_{3}$ & $\mathrm{Fe}_{2} \mathrm{O}_{3}$ & $\mathrm{MgO}$ & $\mathrm{CaO}$ & $\mathrm{Na}_{2} \mathrm{O}_{5}$ & $\mathrm{~K}_{2} \mathrm{O}$ & $\mathrm{P}_{2} \mathrm{O}_{3}$ & $\mathrm{SO}_{3}$ \\
\hline $54.3 \%$ & $8.2 \%$ & $14.3 \%$ & $1.7 \%$ & $10.9 \%$ & $0.7 \%$ & $1.5 \%$ & $3.5 \%$ & $0.8 \%$ \\
\hline
\end{tabular}

Tableau 2. Caractérisation minéralogique et physique des sédiments traités, (SCORDIA et al., 2008)

\begin{tabular}{llllllllll}
\hline $\begin{array}{l}\text { Masse } \\
\text { volumique }\end{array}$ & $\begin{array}{l}\text { Surface } \\
\text { spécifique }\end{array}$ & $\begin{array}{l}\text { Porosité } \\
\text { accessible }\end{array}$ & $p H$ & $\begin{array}{l}\text { Phases } \\
\text { cristallines }\end{array}$ & $\rho_{\text {dOPN }}$ & $w_{O P N}$ & IPI & $W_{L}$ & $V_{B S}$ \\
\hline $2.76 \mathrm{~g} / \mathrm{cm}^{3}$ & $3.77 \mathrm{~m}^{2} / \mathrm{g}$ & $3.5 \%$ & 12.4 & $\begin{array}{l}\text { Quartz, } \\
\text { hématite, } \\
\text { mica }\end{array}$ & $\begin{array}{l}1.33 \\
\mathrm{~g} / \mathrm{cm}^{3}\end{array}$ & $30.6 \%$ & 26 & $50.2 \%$ & 0.03 \\
& & & & & & & & & \\
\end{tabular}

Un deuxième matériau issu des opérations de dragage fluvial, a été utilisé. Il s'agit d'un sable de maçonnerie. La courbe granulométrique de ce dernier a été reportée sur la figure 1. Ce matériau, permet, grâce à sa capacité drainante, de réduire la teneur en eau du mélange composé de sédiments traités, sable, ciment et eau. Le liant hydraulique utilisé est un ciment CEMIII 32.5R produit par la compagnie Calcia. Ce ciment a été utilisé pour étudier à la fois une valorisation des sédiments traités dans des matériaux autocompactants, potentiellement utilisables dans le remplissage des excavations urbaines (il fournit une résistance à court terme élevée), mais aussi pour une potentielle 
utilisation en couche de forme (long terme). Dans certains mélanges, la chaux vive est utilisée afin d'optimiser le coût économique et réduire l'impact environnemental de cette valorisation.

\subsection{Méthodologie expérimentale}

Pour être utilisé comme matériau autocompactant ou routier, les propriétés physiques et mécaniques du matériau doivent satisfaire certains critères. Tout d'abord, le matériau doit présenter un affaissement au cône d'Abrams (Slump) compris entre 16 et $20 \mathrm{~cm}$, caractérisant un matériau fluide. Ce critère est nécessaire pour une éventuelle valorisation dans un matériau autocompactant (utilisation en milieu urbain). Ceci permet de garantir une meilleure pompabilité lors de la mise en œuvre sur chantier. Ensuite, l'indice portant immédiat (IPI) à 24 heures, doit être supérieur 10 (AUTOCAN, 2002), afin de permettre la remise en circulation des engins du chantier sur la zone d'intervention 24 heures après l'achèvement de l'opération de remblayage. Pour assurer une réexcavation assez facile, possible à long terme, manuellement ou avec une mécanisation légère, la valeur de la résistance à la compression à 28 jours doit être comprise entre 0.7 et $2 \mathrm{MPa}$, selon le LCPC-SETRA (1994, 2007). Enfin, la résistance à la traction $\left(\mathrm{R}_{\mathrm{tb}}\right)$ doit excéder $0.25 \mathrm{MPa}$ (LCPC-SETRA, 2007). Dans cette étude $\mathrm{R}_{\mathrm{tb}}$ est déterminée à 28 jours Ce seuil garantit que le matériau a développé une résistance suffisante contre le gel. La résistance à la traction est déterminée à partir du test Brésilien (AFNOR, 2001). Les caractéristiques à atteindre pour l'utilisation en matériau autocompactant sont récapitulées dans le tableau 3.

Tableau 3. Propriétés d'un matériau pour l'utilisation en matériau autocompactant (AUTOCAN, 2002).

\begin{tabular}{llll}
\hline Slump $(\mathrm{cm})$ & $I P I_{24 h}$ & $R_{c 28}(\mathrm{MPa})$ & $R_{t b 28}(\mathrm{MPa})$ \\
\hline $16 \leq$ slump $\leq 20$ & $\geq 10$ & $0.7 \leq R_{c 28} \leq 2$ & $\geq 0.25$ \\
\hline
\end{tabular}

Avec une résistance à la compression Rc plus élevée et une valeur d'IPI plus importante, le matériau valorisé atteint les seuils permettant son utilisation en couche de forme. Ces seuils sont récapitulés dans le tableau 4.

Tableau 4. Propriétés d'un matériau pour l'utilisation en couche de forme (GTS, 2000).

\begin{tabular}{lll}
\hline$I P I$ & $R_{c 28}(\mathrm{MPa})$ & $R_{t b 28}(\mathrm{MPa})$ \\
\hline$\geq 25$ & $\geq 2.5$ & $\geq 0.25$ \\
\hline
\end{tabular}

Dans cette étude, des sédiments traités par le procédé Novosol ${ }^{\circledR}$ et tamisés à $2 \mathrm{~mm}$ et du sable de maçonnerie sont utilisés comme matériaux fins dans la formulation d'un matériau routier et d'un matériau autocompactant. Les liants utilisés sont le Ciment 32.5 
R et la chaux vive. En se basant sur le GTS (2000), différentes formulations ont été étudiées. Tout d'abord, des formulations de base, sans ciment, ont été étudiées, en variant les pourcentages des sédiments traités et du sable : M100-0, M75-25, M50-50 et M25-75 (M\% des sédiments - \% du sable). Ensuite, plusieurs mélanges ont été confectionnés par ajout de différents pourcentages de ciment $(2 \%, 4 \%$ et $7 \%$ de la masse totale des matériaux fins). Pour certains de ces mélanges, l'influence de l'ajout de la chaux vive a été étudiée en variant son pourcentage.

La première étape du protocole expérimental consiste à peser les différents constituants du mélange et à les introduire dans le malaxeur. Dans un premier temps, l'ensemble est homogénéisé. L'eau est introduite avec précaution, en deux temps afin de bien contrôler l'affaissement qui ne devra pas excéder $20 \mathrm{~cm}$, dans le cas où le matériau valorisé est utilisé en matériau autocompactant. Au total, la masse d'eau ajoutée varie entre 20 et $50 \%$ de la masse des matériaux fins utilisés.

L'affaissement du produit à son état frais, est alors déterminé par un cône d'Abrams. D'autre part, l'IPI est mesuré par un essai de poinçonnement. Pour déterminer la résistance à la compression et à la traction, les éprouvettes cylindriques confectionnées sont de dimensions $60 \times 120 \mathrm{~mm}$. Toutes les éprouvettes ont été conservées dans un récipient en plastique bien fermé. Par ailleurs, la répétabilité des essais et la reproductibilité des résultats expérimentaux ont fait l'objet d'un soin particulier.

\section{Analyse des résultats}

\subsection{Détermination de l'indice portant immédiat}

L'IPI ${ }_{24 h}$ permet d'évaluer l'aptitude d'un matériau à supporter la circulation des engins et de vérifier le critère de "traficabilité" au bout de 24 heures. L'indice portant immédiat est déterminé, selon la norme NF P 94-078 (AFNOR, 1997b), par l'essai de poinçonnement. Cet indice est déterminé à 24,48 et 72 heures pour étudier son évolution au cours du temps et pour répondre aux exigences des normes. Pour les mélanges, confectionnés sans la chaux vive, les valeurs de l'IPI ${ }_{24 h}$ enregistrées, sont trop faibles (inférieures à 10) (figure 2). Seul le mélange M 25-75-7 (7\% de ciment) présente une valeur d'IPI ${ }_{24 h}$ supérieure à 10 . Pour valoriser des quantités de sédiments encore plus importantes, tout en économisant les coûts, des améliorations sont à apporter à cette formulation. La figure 3 montre que pour le mélange de formule M-7525-7, la traficabilité n'est permise qu'au bout de 48 heures (IPI $<10$ à 24 heures).

L'influence de l'ajout de différentes quantités de chaux vive au mélange M-75-25-7, $(1 \%, 2 \%$, et $4 \%$ de la masse totale des matériaux fins utilisés) est alors étudiée. La figure 4 montre que l'IPI à 24 heures croit progressivement avec l'augmentation du pourcentage de chaux dans le mélange. Avec $1 \%$ et $2 \%$ de chaux, l'IPI augmente successivement de $20 \%$ et $60 \%$. Ces valeurs restent, néanmoins inférieures à 10 . Pour le mélange confectionné avec 4\% de chaux, l'IPI augmente de 180\% (de l'ordre de 14), 
satisfaisant ainsi le premier critère permettant l'utilisation en matériau autocompactant. Ainsi, un pourcentage de $4 \%$, de chaux vive, sera retenu pour la formulation finale du matériau autocompactant et potentiellement en couche de forme.

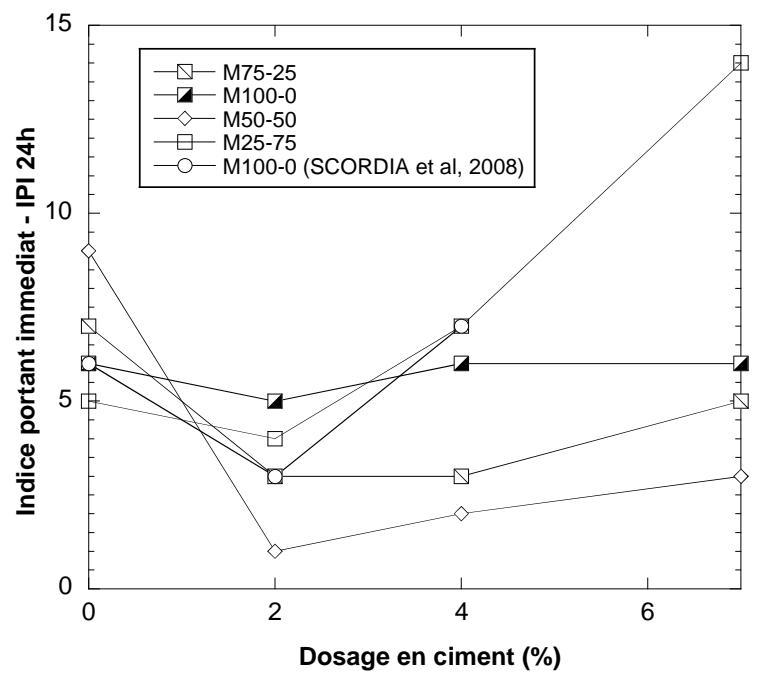

Figure 2. L'IPI à 24 heures des mélanges de base en fonction du dosage en ciment.

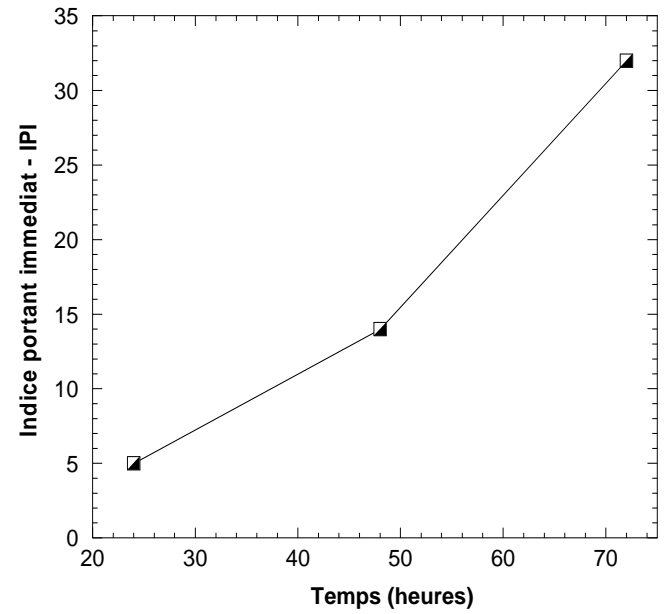

Figure 3. Evolution de l'indice portant immédiat au cours du temps du mélange M 75-25-7.

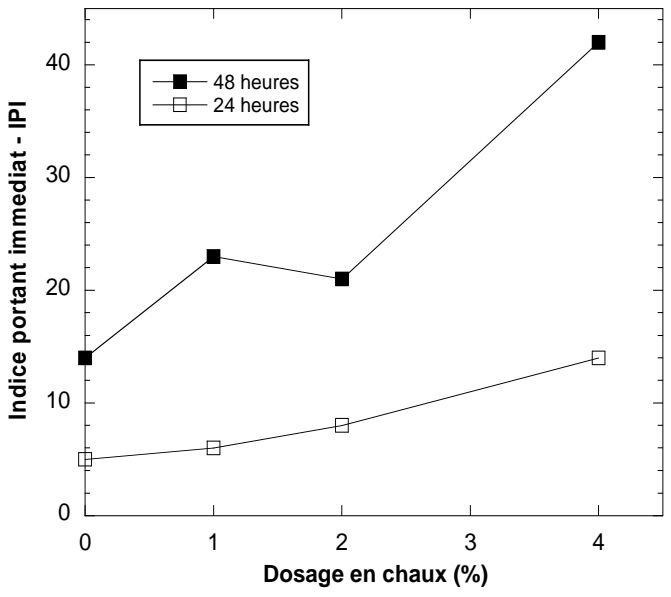

Figure 4. L'indice portant immédiat en fonction du dosage en chaux vive $d u$ mélange M 75-25-7.

4.2 Résistance à la compression et à la traction à 28 jours

La résistance à la compression $\mathrm{R}_{\mathrm{c} 28}$ a été déterminée par une presse de compression uniaxiale sur des éprouvettes $60 \times 120 \mathrm{~mm}$ selon la norme NF P 94-077 (AFNOR, 1997a). Les résultats des formulations de base sont donnés par la figure 5. Pour atteindre une résistance à la compression $\mathrm{Rc}_{28}$ comprise entre $0.7 \mathrm{MPa}$ et $2 \mathrm{MPa}$, le dosage en ciment doit être au moins égal à $4 \%$. Par mesure de sécurité, et pour tenir compte des aléas de la mise en place sur chantier, il est préférable de se situer aux 
alentours de 1.5 MPa dans le cas d'une valorisation en matériau autocompactant (LCPC-SETRA, 2007).

D'autre part, la résistance à la traction doit être supérieure à $0.25 \mathrm{MPa}$. Les valeurs de $\mathrm{Rtb}_{28}$ en fonction du dosage en ciment, représentées sur la figure 6, montrent qu'un dosage en ciment au moins égal à $4 \%$ est nécessaire, sauf pour la formulation M100-0 $\left(\mathrm{Rtb}_{28}<0.25 \mathrm{MPa}\right)$. Ainsi, les formulations satisfaisant les critères mécaniques à tenir en compte, dans le cas d'une valorisation en matériau autocompactant sont M75-25-4 et M50-50-4. Il est plus judicieux de choisir la première qui nécessite des quantités de sédiments plus importantes.

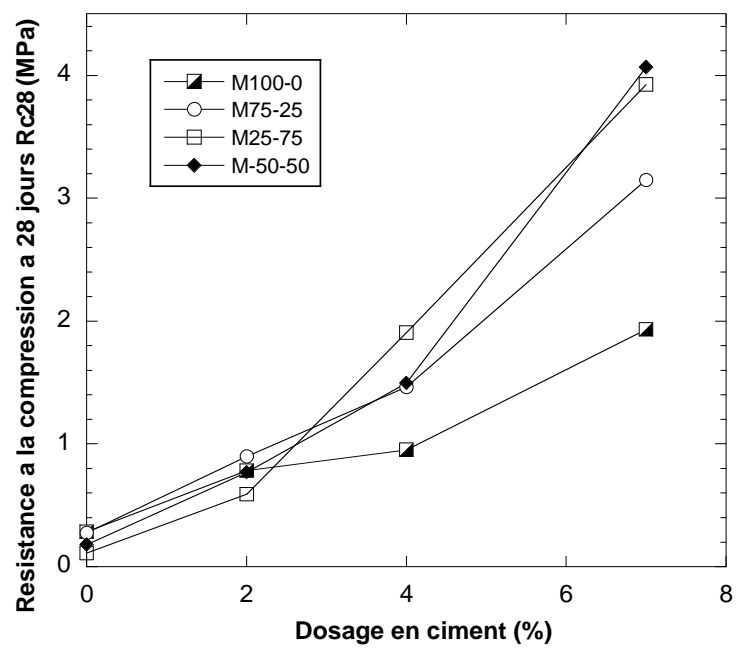

Figure 5. Evolution de $R c_{28}$ en fonction $d u$ dosage en ciment des mélanges de base.

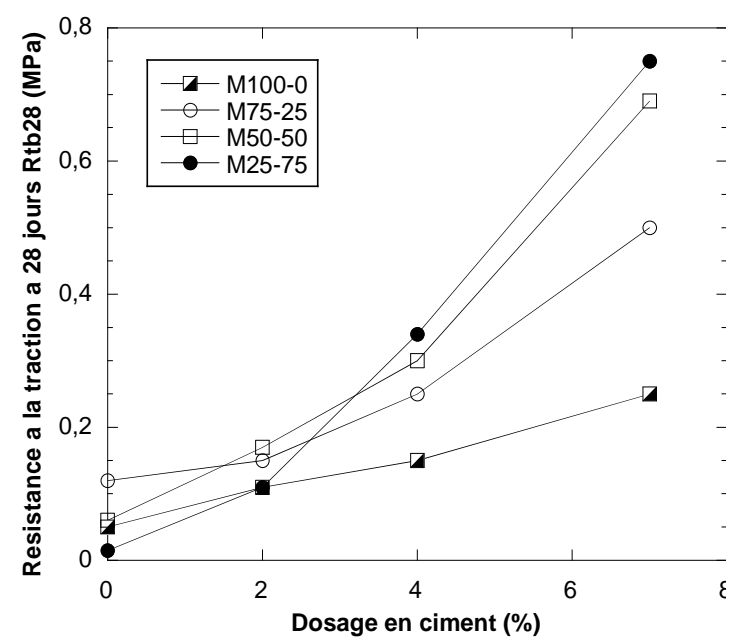

Figure 6. Evolution de $\mathrm{Rtb}_{28}$ en fonction du dosage en ciment des mélanges de base.

Pour optimiser le dosage de la formulation à retenir, l'influence de l'ajout de 1, 2 et 4\% de chaux vive au mélange M75-25-7 a été étudiée. Les résultats obtenus sont présentés dans la figure 7. On observe une amélioration considérable de $\mathrm{Rc}_{28}$ et de $\mathrm{Rtb}_{28}$ en fonction du dosage en chaux. Ainsi, un pourcentage de ciment de l'ordre de $2 \%$ avec $4 \%$ de chaux vive est suffisant pour atteindre les seuils réglementaires que doit satisfaire un matériau autocompactant.

La formulation retenue se compose donc de $75 \%$ de sédiments traités, $25 \%$ de sable de maçonnerie, $2 \%$ de ciment et $4 \%$ de chaux. L'indice portant immédiat mesuré, pour cette formulation, est égal à 16 à 24 heures et 33 à 48 heures. La résistance à la compression est de l'ordre de 4.25 MPa. Les résultats obtenus sont regroupés dans le Tableau 5. 
Valorisation des sédiments fluviaux traités en vue d'une utilisation en génie civil : 5.9

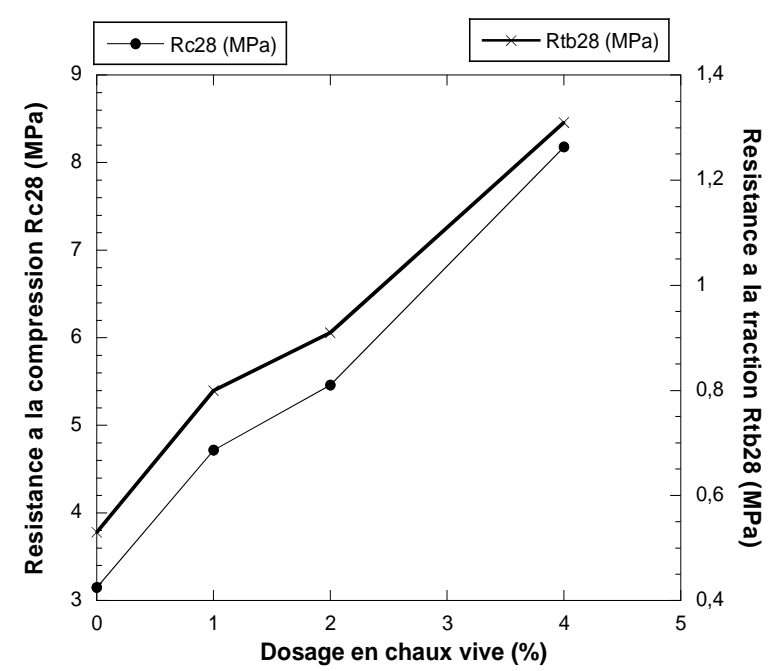

Figure 7. Evolution de $R_{c 28}$ et $R_{t b 28}$ en fonction du dosage en chaux du mélange M75-25-7.

Tableau 5. Résultats expérimentaux obtenus pour la formulation M75-25-2-4.

\begin{tabular}{lllllllll}
$I P I_{24 h}$ & $I P I_{48 h}$ & $w_{0}$ & $w_{24 h}$ & $w_{48 h}$ & $w_{28 j}$ & $R c_{28}$ & $R_{t b 28}$ & $E_{28}$ \\
\hline 16 & 33 & $35.9 \%$ & $29.5 \%$ & $28.0 \%$ & $21.7 \%$ & $4.25 \mathrm{MPa}$ & $0.758 \mathrm{MPa}$ & $10.82 \mathrm{GPa}$ \\
\hline
\end{tabular}

La même formulation est retenue pour la valorisation en couche de forme, sous la réserve de retarder la circulation 48 heures après l'achèvement des travaux. A partir de la valeur de $\mathrm{Rt}(\mathrm{Rt}=0.8 \mathrm{Rtb})$ et du module d'Young E à 28 jours, déterminés sur des éprouvettes de dimensions $100 \times 100 \mathrm{~mm}$, le matériau de la formulation retenue est positionné sur l'abaque de situation des matériaux de couche de forme traités aux liants hydrauliques (GTS, 2000) (figure 8). Le matériau obtenu appartient à la zone 3, qui se caractérise par un intérêt technique et économique pour le traitement des sols.

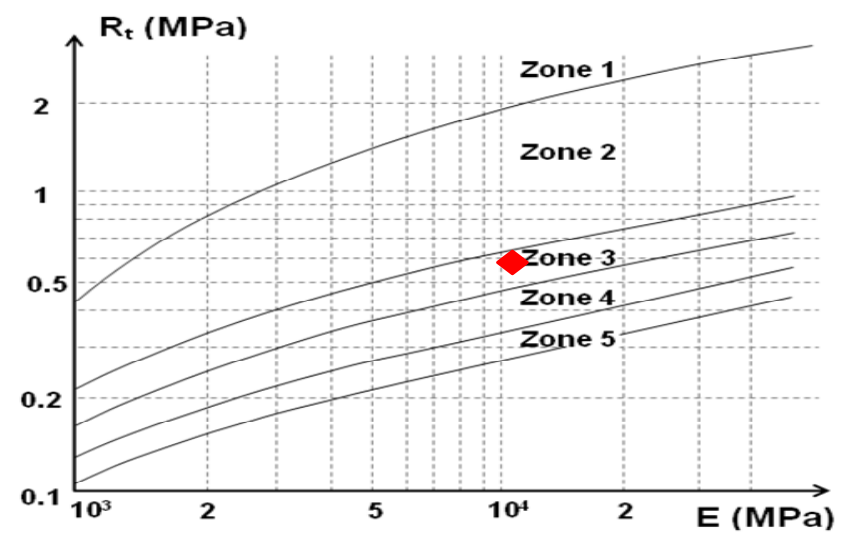

Figure 8. Position du mélange retenu sur l'abaque de situation des matériaux de couche de forme (GTS, 2000). 


\section{Conclusion}

L'objectif de cet article est d'étudier la faisabilité d'une valorisation, après traitement, des sédiments pollués, dans des matériaux de génie civil notamment les matériaux autocompactants pour un usage en milieu urbain ou en couche de forme pour un usage en matériau routier. Pour cela une campagne expérimentale a été réalisée. Cette dernière a permis de retenir une formulation d'un matériau potentiellement valorisable en génie civil, dont $75 \%$ sont des sédiments traités répondant ainsi à la problématique du volume. Les mélanges étudiés, sans ou avec ciment ou/et chaux, en variant les pourcentages des différents constituants du produit ont permis de mettre en évidence l'influence de chaque constituant. En effet, l'ajout du ciment CEM III 32.5R et de la chaux vive fut nécessaire pour améliorer les principales propriétés notamment l'IPI ${ }_{24 h}, I_{48} I_{48}, R_{28}$ et $\mathrm{Rtb}_{28}$ et atteindre les seuils réglementaires. Pour le mélange M75-25-7, constitué de 7\% de ciment, sans chaux, la traficabilité n'est permise qu'au bout de 48 heures, puisque l'IPI24h est inférieur au seuil réglementaire. Cette formulation peut être retenue sous la contrainte de retarder la remise en circulation des engins. L'ajout de $4 \%$ de chaux vive à ce mélange, a permis de pallier cette contrainte, dans le cas d'un matériau autocompactant, en augmentant la valeur de $1^{\prime} \mathrm{IPI}_{24 \mathrm{~h}}$ de $180 \%$ par rapport à celle d'un mélange sans chaux. L'utilisation d'autres liants hydrauliques mieux appropriés pourraient améliorer ce résultat. Finalement, la formulation retenue pour la valorisation en matériau autocompactant, est celle qui contient du ciment et de la chaux avec $2 \%$ de ciment, $4 \%$ de chaux, $75 \%$ de sédiments traités passant au tamis $2 \mathrm{~mm}$ et $25 \%$ de sable. Cette formulation est également retenue pour la valorisation en couche de forme, sous la réserve de retarder la circulation à 48 heures après l'achèvement des travaux. En incorporant $75 \%$ de sédiments, cette solution permet de valoriser des volumes assez importants de sédiments traités et présente ainsi un grand intérêt économique et environnemental. Des essais complémentaires doivent être menés afin de caractériser la durabilité et l'impact environnemental, du nouveau produit. Les résultats de cette étude sont très encourageants et montrent que de nouvelles voies de valorisation des sédiments de dragage traités par le procédé Novosol ${ }^{\circledR}$ dans les matériaux routiers sont pleinement envisageables.

\section{Références bibliographiques}

AFNOR (1997a). NF P 94-077. Sols : Reconnaissance et Essais - Essai de compression uniaxiale.

AFNOR (1997b). NF P 94-078. Sols : Reconnaissance et essais. Indice CBR après immersion. Indice CBR immédiat. Indice Portant immédiat. Mesure sur échantillon compacté dans le moule CBR.

AFNOR (1998). XP X 31-210. Déchets - Essais de lixiviation.

AFNOR (2001). NF P 98 232-3. Essais relatifs aux chaussées - détermination des caractéristiques mécaniques des matériaux traités aux liants hydrauliques - Partie 3 
Valorisation des sédiments fluviaux traités en vue d'une utilisation en génie civil : 5.11

essai de compression diamétrale sur matériaux traités aux liants hydrauliques et pouzzolaniques.

AGOSTINI F., SKOCZYLAS F., LAFHAJ Z. (2007). About a possible valorisation in cementitious materials of polluted sediments after treatment. Cement and Concrete Composites, Vol. 29, n 4, pp 270-278. doi:10.1016/j.cemconcomp.2006.11.012

ALZIEU C. (1999). Dragages et Environnement Marin: état des connaissances. Editions Ifremer, Plouzanée, 209 p.

AUBERT J.E. (2002). Valorisation d'une cendre d'incinérateur d'ordures ménagères, traitée par le procédé REVASOL ${ }^{\circledR}$, dans le béton hydraulique. Thèse de doctorat, Université Paul Sabatier de Toulouse.

AUTOCAN (2002). Autocan-Procédé pour remblayage de tranchées. Eurovia, 4 p.

BRAKNI S., ABRIAK N.E., GREGOIRE P., ZENTAR R. (2007). Valorisation de boues de dragage maritime en granulats artificiels en vue de stabiliser l'érosion littorale. $25^{\mathrm{e}}$ rencontre de l'AUGC, 23-25 mai 2007, Bordeaux, 9 p.

BOUTOUIL M., LEVACHER D. (2000). Traitement et valorisation des vases de dragages par solidification/stabilisation: état de l'art. VI ${ }^{\mathrm{e} m e s}$ Journées Nationales Génie Civil - Génie Côtier, Caen, France, pp 367-375. doi:10.5150/jngcgc.2000.031-B

CRANNELL B.S., EIGHMY T.T., KRZANOWSKI J.E., EUSDEN J.D., SHAW E.L., FRANCIS C.A. (2000). Heavy métal stabilization in municipal solid waste combustion bottom ash using soluble phosphate. Waste Management, Vol. 20, pp 135-148. doi:10.1016/S0956-053X(99)00312-8

DUAN Z., LEVACHER D., SANCHEZ M. (2008). Comportement d'une couche de sédiment de dragage en séchage naturel. $\mathrm{X}^{\mathrm{èmes}}$ Journées Nationales Génie Côtier Génie Civil, Sophia Antipolis, pp 709-718. doi:10.5150/jngcgc.2008.067-D

DUBOIS V., ABRIAK N. E., ZENTAR R., BALLIVY G. (2009). The use of marine sediments as a pavement base material. Waste Management, Vol. 29, pp 774-782. doi:10.1016/j.wasman.2008.05.004

GTS (2000). Traitement des sols à la chaux et aux liants hydrauliques-Guide Technique, LCPC-SETRA, $240 \mathrm{p}$.

GTR (2000). Guide Technique pour la réalisation des remblais et des couches de forme. Fascicule I \& II, Editions du Setra-LCPC, 200 p.

KAMALI S., BERNARD F., ABRIAK N.E., DEGRUGILLIERS P. (2008). Marine dredged sediments as new materials resource for road construction. Waste Management, Vol. 28, pp 919-928. doi:10.1016/j.wasman.2007.03.027

KRIBI S., NZIHOU A., SHARROCK P. (2004). Stabilization of heavy metals from dredged sediment. Tailoring of residue properties. In: VAZQUEZ E, HENDRIKS CHF, JANSSEN GMT. Editors. Proceedings of the international RILEM conference, Barcelona, Spain, 8-11 November 2004, Vol. 2. RILEM Publications; 2004, ISBN 2912143-52-7, $1168 \mathrm{p}$. 
LAFHAJ Z., SALICETO A., COHEN-SOLEL L., COUDRAY Y., HUYNH T.T., LEGUEN B., ANGOUNI F. (2007). The use of the Novosol ${ }^{\mathbb{R}}$ process for the treatment of polluted marine sediment. Journal of Hazardous Materials, Vol. 148, $\mathrm{n}^{\circ} 3$, pp 606-612. doi:10.1016/j.jhazmat.2007.03.029

LAFHAJ Z., SAMARA M., AGOSTINI F., BOUCARD L., SKOCZYLAS F., DEPELSENAIRE G. (2008). Polluted river sediments from the north region of France: treatment with Novosol ${ }^{\circledR}$ process and valorization in clay bricks. Construction \& Building Materials. Vol. 22, n 5, pp. 755-762. doi:10.1016/j.conbuildmat.2007.01.023

LAPERCHE V., TRAINA S.J., GADAM P., LOGAN T.J. (1996). Chemical and mineralogical characterizations of $\mathrm{Pb}$ in a contaminated soil: reactions with synthetic apatite. Environ Sci Technol ; Vol. 30, pp 3321-3326. doi:10.1021/es960141u

LCPC-SETRA (1994). Remblayage des tranchées et réfection des chaussées. Guide technique, mai 1994, $80 \mathrm{p}$.

LCPC-SETRA (2007). Remblayage des tranchées et réfection des chaussées. Compléments au guide technique Setra-LCPC de mai 1994, CETE Normandie - Centre, $10 \mathrm{p}$.

LONDON CONVENTION (1972). Convention on the prevention of marine pollution by dumping of wastes and other matter. 1972, London.

NGUYEN T.B. (2008). Valorisation de sédiments traités par le procédé Novosol ${ }^{\circledR}$ dans les matériaux routiers. Thèse de Doctorat, Université de Toulouse.

Official Journal J.O.No. 184 (10 August 2000), 12415 (NOR: ATEE0090254A).

SOLVAY (2002). Publication EP1341728 (19/04/2002). Patent correspondant : FR2815338 (17/10/2000). In: Procédé d'inertage de boues, Solvay.

SAMARA M., LAFHAJ Z., CHAPISEAU C. (2008). Valorization of stabilized river sediments in fired clay bricks: factory scale experiment. Journal of Hazardous Material, Vol. 163, pp 701-710. doi:10.1016/j.jhazmat.2008.07.153

SCORDIA P.Y., LAFHAJ Z., SKOCZYLAS F., MONGOUIN T. (2008). Characterization and valorization of polluted and treated river sediments in road. European Journal of Environmental and Civil Engineering. Vol. 7, n 4, pp 451- 469.

USEPA - United States Environmental Protection Agency- (1986). Test Methods for Evaluating Solid waste : Physical/Chemical Methods. SW-846. Washington, DC.

USEPA - United States Environmental Protection Agency- (1992). EPA Test Method 1311: TCLP -Toxicity Characteristic Leaching Procedure-. Washington, DC.

WENG C.H., LIN D.F., CHIANG P.C. (2003). Utilization of sludge as brick materials. Adv. Environ. Res. Vol. 7, pp 679-685. doi:10.1016/S1093-0191(02)00037-0

ZENTAR R., DUBOIS V., ABRIAK N.E. (2008). Mechanical behaviour and environmental impacts of a test road built with marine dredged sediment. Resources, Conservation and recycling, Vol. 52, pp 947-954. doi:10.1016/j.resconrec.2008.02.002 
Valorisation des sédiments fluviaux traités en vue d'une utilisation en génie civil : 5.13

\title{
Valorisation of treated river sediments in civil engineering works
}

\author{
Zhibo DUAN $^{1}$, Zoubeir LAFHAJ ${ }^{1}$, \\ Imen BEL HADJ ALI ${ }^{1}$, Sébastien DUCELLIER ${ }^{1}$
}

1. Laboratoire de Mécanique de Lille, CNRS UMR 8107, Boulevard Paul Langevin, 59655, Villeneuve d'Ascq Cedex, France.

Ecole Centrale de Lille, Cité Scientifique, 59651, Villeneuve d'Ascq Cedex, France. Zoubeir.lafhaj@ec-lille.fr

Translated version not provided by the authors

Version traduite non fournie par les auteurs 
5.14 : Revue Paralia - Vol. 6 (2013) 